\title{
Atividades de controle de Aedes aegypti em municípios da Região Sul do Estado de São Paulo
}

\section{Control activities of Aedes aegypti in counties of the south region of São Paulo State}

\author{
Maria de Fátima Domingos ${ }^{1}$, Cleide Dantas de Oliveira ${ }^{1}$, Gerson Laurindo Barbosa ${ }^{1}$, Dalva Marli Valério Wanderley ${ }^{1}$ \\ 1. Doutora em Epidemiologia pela Faculdade de Saúde Pública da Universidade de São Paulo. Superintendência de Controle de Endemias - SUCEN \\ - SES - SP, Brasil.
}

\section{Resumo}

Introdução: Neste estudo, são analisadas as atividades de vigilância e controle do Aedes aegypti, vetor da dengue, executadas por municípios selecionados da região do Litoral Sul do Estado de São Paulo. Método: Análise de dados secundários extraídos do programa informatizado referente às atividades de vigilância e controle do Aedes aegypti. Resultados: Índice de Infestação Predial: foram realizadas 31 medidas, com valores médios inferiores a 2,0; Casa-a-casa: as maiores coberturas foram observadas nos segundos quadrimestres de cada ano, devido ao comprometimento das equipes com a transmissão no primeiro semestre; Pontos Estratégicos: houve redução do número de imóveis trabalhados nos últimos quatro anos, positividade média foi de 33,8\% com tendência de aumento; Imóveis Especiais: registrou-se diminuição no número de imóveis trabalhados nos últimos dois anos, e a positividade média foi de $24,7 \%$. Conclusão: Verificaram-se baixas coberturas das atividades previstas na norma técnica, o que pode favorecer a manutenção da infestação. Não foi possível constatar a implantação das orientações técnicas a partir dos resultados dos índices de infestação para subsidiar o emprego de medidas de controle entomológico.

Palavras-chave: Aedes aegypti. Dengue. Controle de insetos. Vigilância epidemiológica.

\begin{abstract}
Introduction: In this study, the activities of surveillance and control of Aedes aegypti, the dengue vector, are analyzed, as carried out by selected municipalities in the Southern Coast of São Paulo State. Method: Analysis of secondary data extracted from computerized program activities related to the surveillance and control of Aedes aegypti. Results: Infestation Index Building: 31 measurements were performed, with average values below 2.0; house-to-house: the largest coverage was observed in the second four-month periods of each year due to the commitment of the teams with the transmission in the first semester, Strategic Buildings: there was a reduction in the number of properties worked in the past four years, average positivity was $33.8 \%$ with an upward trend; Special Buildings: there was a decrease in the number of properties worked in the last two years, and the average positivity was $24.7 \%$. Conclusion: There was low coverage of the activities as foreseen in the standard techniques, what may promote the maintenance of infestation. It was not possible to verify the implementation of technical guidance from the results of infestation to subsidize the use of measurements of entomological control.
\end{abstract}

Keywords: Aedes aegypti. Dengue Virus. Insect Control. Epidemiological Surveillance.

\section{INTRODUÇÃO}

O combate ao Aedes aegypti foi institucionalizado no Brasil de forma sistemática a partir do início do século XX, durante as epidemias de febre amarela urbana ${ }^{1}$. Nas décadas de 1950 e 1960, o Brasil e outros 17 Países das Américas conseguiram eliminar o Aedes aegypti, utilizando estratégia de campanha nacional, verticalizada, com estrutura militar, marcada pela disciplina e hierarquia. Como alguns países vizinhos não obtiveram êxito, o Brasil sofreu várias reinfestações, precocemente detectadas e eliminadas ${ }^{2}$. Nesse país, as condições socialmente favoráveis à expansão do vetor possibilitaram sua dispersão desde a reintrodução em 1976, que não conseguiu ser controlada com métodos tradicionais usados no combate aos vetores das doenças ${ }^{3}$.

No Estado de São Paulo, o Aedes aegypti foi detectado no início na década de 1980, no Porto de Santos, quando foram empregadas medidas de controle que bastaram 
para a sua eliminação. Até 1984, o Ministério da Saúde foi o único responsável pelo controle de Aedes aegypti no Estado de São Paulo, onde desenvolvia regularmente atividades em portos e aeroportos internacionais, além de realizar, esporadicamente, atividades em outras áreas do Estado. No ano de 1985, a Superintendência de Controle de Endemias (Sucen), da Secretaria de Saúde do Estado de São Paulo, realizou uma investigação entomológica em todos os municípios paulistas e detectou focos do Aedes aegypti em 30 deles, localizados a oeste do Estado, dos quais nove apresentavam o vetor estabelecido na área urbana. A partir de então, a vigilância entomológica foi mantida na totalidade dos municípios e permitiu acompanhar a expansão geográfica do vetor de forma contínua e rápida, do oeste para o leste do Estado, com distribuição limitada a áreas urbanas e aglomerados rurais ${ }^{4}$.

Na região do litoral sul do Estado, a infestação por Aedes aegypti foi registrada em 1995, no município de Santos, onde também ocorreu a primeira transmissão de dengue em 1997, representando um grande problema de saúde pública, pois a região apresenta características bastante propícias ao estabelecimento do vetor e da doença, relacionadas ao clima, à ocupação do solo, e à economia, com destaque para o Porto de Santos, ao polo petroquímico e ao turismo ${ }^{5}$. De fato, em 1998, a doença já se apresentava nos municípios de Cubatão, Guarujá, Praia Grande e São Vicente, onde se estabeleceu. A série histórica de transmissão destaca picos epidêmicos nos anos de 2001, 2002 e 2010, quando foram notificados 20.024, 26.989 e 35.303 casos respectivamente, assim como a importante contribuição da transmissão de dengue desses municípios para a situação epidemiológica no Estado de São Paulo: entre 1997 e 1999, cerca de 50\%, e em 2002, 68,9\% dos casos confirmados da doença foram contraídos nesses municípios ${ }^{6}$.

Em 1997, com a instituição do Plano de Erradicação do Aedes aegypti (PEAa); do Ministério da Saúde (MS), foi iniciado o processo e a implantação das ações de controle vetorial por parte dos municípios, que passaram a receber recursos financeiros por meio de convênios, direcionados à construção de estrutura para a execução das atividades de vigilância e controle do vetor de forma sistematizada ${ }^{1}$. Essa forma de repasse de recursos foi substituída a partir de 2000 com a publicação da Portaria 13997, que definiu as competências da União, dos estados, dos municípios e do Distrito Federal, na área de epidemiologia e controle de doenças, e a sistemática de financiamento, posteriormente substituída pelas Portarias $1172^{8}, 3252^{9}$ e atualmente, $1378^{10}$.

No ano de 2002, já com a meta oficial de erradicação do vetor abandonada, o Ministério da Saúde implantou o Programa Nacional de Controle da Dengue (PNCD) com os objetivos de reduzir a infestação por Aedes aegypti, a incidência da dengue e a letalidade por febre hemorrágica de dengue ${ }^{3}$. O PNCD identificou um conjunto de municípios que apresentavam situação epidemiológica diferenciada na transmissão de dengue, denominando-os de prioritários, selecionados à época pelos seguintes critérios:

- ser capital de estado ou pertencer à sua região metropolitana;

- ter população igual ou superior a 50.000 habitantes;

- ser receptivo à introdução de novos sorotipos de dengue (fronteiras, portuários, núcleos de turismo, etc.).

No Estado de São Paulo, os municípios de Cubatão, Guarujá, Praia Grande, Santos e São Vicente, localizados no litoral sul, foram incluídos nessa classificação e passaram, assim como os demais, a ser sistematicamente acompanhados em suas atividades ${ }^{11}$.

Em 2009, o Ministério da Saúde lançou novas diretrizes para o controle de dengue, contemplando orientações para a revisão dos planos municipais de controle de dengue com incorporação da experiência acumulada nos diversos eixos de atuação: vigilância, assistência, controle de vetores e comunicação social ${ }^{12}$.

No âmbito do Estado de São Paulo, no ano de 2002 foram readequadas as normas de vigilância e controle de Aedes aegypti, ocasião em que foram introduzidas entre as atividades rotineiras de visita casa-a-casa, pesquisa/ tratamento de Pontos Estratégicos (PEs) e levantamento de índices de infestação, os quais os municípios vinham realizando, a pesquisa em Imóveis Especiais (IEs), ou seja, aqueles que, pela grande circulação de pessoas, podem contribuir para a disseminação do vírus em uma área ${ }^{13}$. Essa norma foi atualizada em 2005, sendo mantida a classificação em municípios prioritários, e as atividades de rotina de controle e vigilância vetorial ficou sob responsabilidade municipal ${ }^{11}$. A atividade casa-a-casa consiste na visita domiciliar ao conjunto dos imóveis, excluindo-se os pontos estratégicos e os imóveis especiais. A norma técnica preconiza para um ano de trabalho, um ciclo quadrimestral seguido de um retorno à área mais infestada (intensificação) durante dois meses com tratamento mecânico e/ou químico (larvicida) dos focos encontrados e outro ciclo quadrimestral seguido de nova intensificação. Pontos Estratégicos são imóveis que apresentam grandes quantidades de recipientes em condições favoráveis à proliferação de larvas de Aedes aegypti e, portanto, podem contribuir de forma importante nos níveis de infestação. A norma técnica recomenda a pesquisa do imóvel e o tratamento focal (aplicação de larvicida nos criadouros com larvas) e/ou perifocal (aplicação de produto químico com equipamento de pulverização nos criadouros e ao seu redor) quando do encontro de larvas de Aedes aegypti, com periodicidade mínima quinzenal. Os Imóveis Especiais são aqueles não residenciais de médio e grande porte que apresentam maior importância na disseminação do vírus da dengue em situações de transmissão da doença, em função do grande fluxo e/ou permanência de pessoas e, além disso, a complexidade das edificações favorecem a proliferação 
do vetor. A norma técnica recomenda a vistoria por equipe responsável pelo imóvel com periodicidade trimestral ou semestral, e aplicação de larvicida quando necessário.

O objetivo do presente estudo foi analisar as atividades de vigilância e controle do vetor da dengue executadas pelos municípios do litoral sul do Estado de São Paulo.

\section{MATERIAL E MÉTODO}

Os municípios de Cubatão, Guarujá, Praia Grande, Santos e São Vicente, objeto deste estudo, aqui denominados municípios selecionados, somam 1.288.237 habitantes ${ }^{14}$. A partir de 1998, todos foram considerados infestados por Aedes aegypti, com transmissão autóctone de dengue estabelecida, e inseridos entre os prioritários do $\mathrm{PNCD}^{3}$. Estão localizados no litoral do Estado de São Paulo (Figura 1), onde, juntamente com Bertioga, Itanhaém, Mongaguá região, em 2010, era de 1, 7 milhão de habitantes, e seu grau de urbanização de $99,8 \%{ }^{16}$.

As principais atividades econômicas da região estão baseadas no Porto de Santos, o maior da América do Sul, que engloba uma série de atividades e serviços; no polo industrial de Cubatão, composto por indústrias de grande porte dos setores de produção de petroquímicos, químicos e siderúrgicos; e, no turismo, presente em todos os municípios e responsável pela atração de um grande contingente populacional flutuante, nos períodos de férias e feriados ${ }^{16}$.

Para esse conjunto de cinco municípios selecionados, Cubatão, Guarujá, Praia Grande, Santos e São Vicente, serão apresentadas as informações das atividades de rotina, aplicadas nos imóveis, objetivando à vigilância e ao controle do vetor, sendo que elas devem ser executadas com periodicidade definida, conforme o Programa Estadual de Vigilância e Controle da

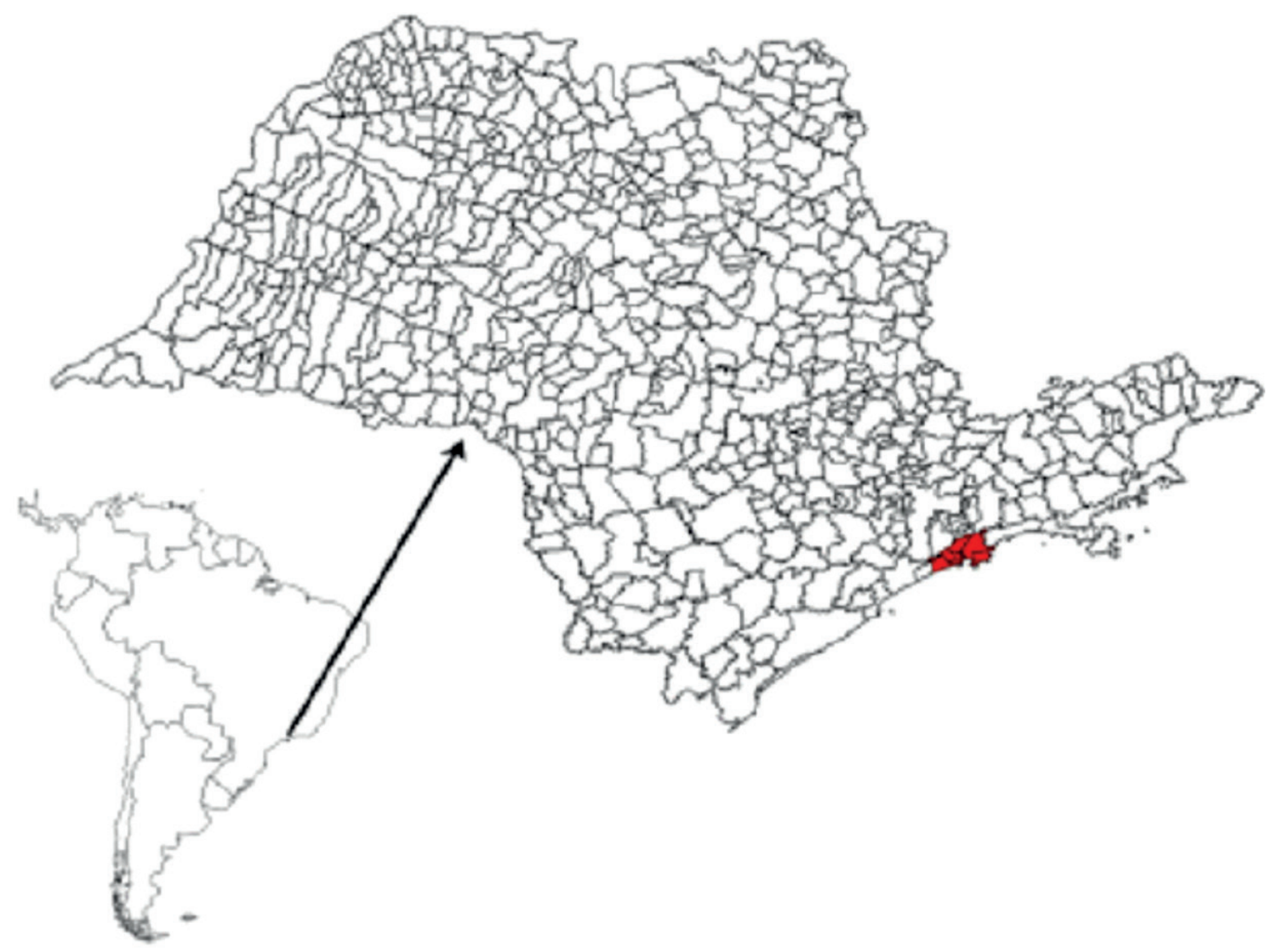

Figura 1. Localização dos municípios selecionados do Litoral Sul do Estado de São Paulo.

e Peruíbe, compõem a região da Baixada Santista, que possui $65 \mathrm{~km}$ contínuos de extensão litorânea e é formada por duas grandes unidades morfológicas: escarpas da Serra do Mar e planície litorânea, de modo que suas cidades encontram-se limitadas, de um lado pela Serra do Mar e, de outro, pelo Oceano Atlântico ${ }^{16}$. Apresenta clima tropical úmido ${ }^{15}$. A população da
Dengue $^{17}$.

Para o cálculo da infestação de PE, foi considerado o percentual de imóveis positivos para Aedes aegypti entre os pesquisados. A porcentagem de tratamento químico desses imóveis considerou o tratamento focal com larvicida de ação residual, no caso, o Bacillus thuringiensis israelensis (Bti) e o diflubenzuron (a partir 
de 2011); o tratamento perifocal com fenitrothion. Para o cálculo da infestação de IE, foi considerado - percentual de imóveis positivos para Aedes aegypti entre os pesquisados. O número de imóveis programados foi calculado utilizando-se o cadastro de imóveis de cada município, excluídos o percentual de pendência representada pelos imóveis fechados, importante nessa região ${ }^{18} \mathrm{e}$ aqueles localizados acima do primeiro andar em edifícios residenciais. O Índice de Infestação Predial (IP) é o percentual de imóveis com larvas de Aedes aegypti entre os pesquisados ${ }^{11,12}$.

A partir de 2010, foi finalizada a fase de implantação das normas operacionais ${ }^{11}$, que introduziram mudanças na periodicidade e na programação do casa-a-casa/ intensificação e na realização dos levantamentos larvários. Os períodos de análise dessas atividades foram 2010 a 2012 e 2009 a 2012, respectivamente. Para PE e IE, o período analisado considerou os anos de 2002 a 2012 e 2005 a 2012, respectivamente.

Foram utilizados como fonte de informações os sistemas informatizados entomológicos (Sisaweb e Aedesw/ Sisaedw) desenvolvidos pela Superintendência de Controle de Endemias-SUCEN, que são sistematicamente alimentados pelos municípios.

O presente estudo foi realizado com base em dados secundários. Seus resultados foram analisados em conjunto e apresentados de forma agregada, de maneira que nenhum dos resultados encontrados foi expresso individualmente, garantindo, dessa forma, o sigilo das informações. Ele foi aprovado pela Superintendência de Controle de Endemias - SUCEN.

\section{RESULTADOS}

Os levantamentos de informações para o cálculo do Índice de Infestação Predial (IIP), ou seja, percentual de imóveis positivos entre os pesquisados, apontaram para este período 9 avaliações nos meses de janeiro, 12 em julho e 20 em outubro, com valores médios nesses meses inferiores a 2,0 (Figura 2).

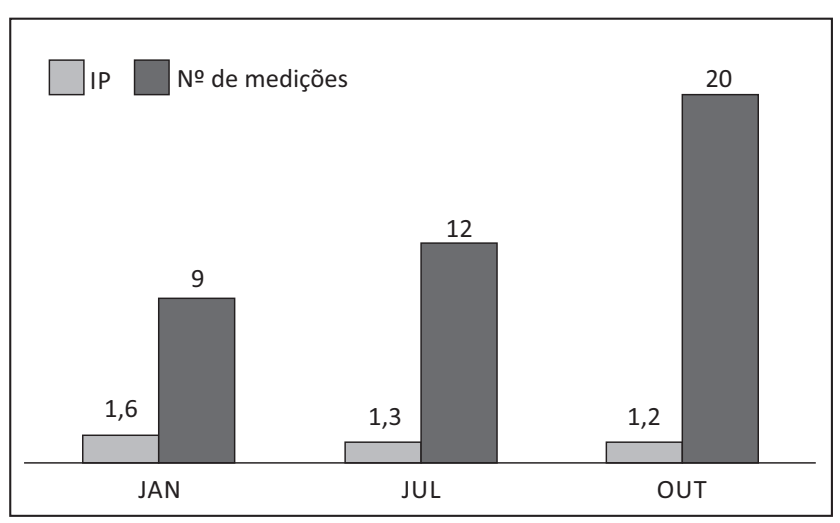

Figura 2. Número de avaliações e média do Índice Predial para Aedes aegypti nos meses de janeiro, julho e outubro. Municípios selecionados do Litoral Sul do Estado de São Paulo, 2009 a 2012.

As atividades desenvolvidas nas visitas casa-a-casa e a intensificação nos cinco municípios podem ser observadas na Tabela 1. Observa-se que as coberturas foram maiores nos segundos quadrimestres dos anos de 2010 e 2011, porém, o mesmo fato não se verificou em 2012. O trabalho de visita a imóveis na atividade de intensificação dirigida para áreas mais problemáticas apontou, para os anos de 2010 a 2011, uma média de 110.000 imóveis nos dois

Tabela 1. Imóveis trabalhados e \% de cobertura em Rotina de casa/casa e trabalhados na intensificação seguindo período. Municípios selecionados do Litoral Sul do Estado de São Paulo, 2010 a 2012.

\begin{tabular}{|c|c|c|c|c|c|c|c|}
\hline \multirow{3}{*}{ Ano } & \multirow{3}{*}{$\begin{array}{c}\text { Imóveis } \\
\text { Programados }\end{array}$} & \multicolumn{4}{|c|}{ Rotina } & \multicolumn{2}{|c|}{ Intensificação } \\
\hline & & \multicolumn{2}{|c|}{ Jan-Abr } & \multicolumn{2}{|c|}{ Jul-Out } & \multirow{2}{*}{$\begin{array}{l}\text { Mai-Jun } \\
\text { Imóveis } \\
\text { Trabalhados }\end{array}$} & \multirow{2}{*}{$\begin{array}{c}\text { Nov-Dez } \\
\text { Imóveis } \\
\text { Trabalhados }\end{array}$} \\
\hline & & $\begin{array}{l}\text { Imóveis } \\
\text { Trabalhados }\end{array}$ & $\%$ Cobertura & $\begin{array}{c}\text { Imóveis } \\
\text { Trabalhados }\end{array}$ & $\%$ Cobertura & & \\
\hline 2010 & 234307 & 122464 & 52.3 & 334028 & 142.6 & 110033 & 114984 \\
\hline 2011 & 234307 & 173778 & 74.2 & 222630 & 95.0 & 120650 & 108761 \\
\hline 2012 & 234307 & 191979 & 81.9 & 123127 & 52.5 & 46361 & 65654 \\
\hline
\end{tabular}


bimestres de 2010 e 2011, com redução para uma média de 56.000 imóveis nos dois bimestres de 2012.

No que diz respeito à pesquisa nos imóveis cadastrados como Pontos Estratégicos (PE), a observação do trabalho, a partir de 2002, apontou uma diminuição do número dos que foram trabalhados no último quadriênio em comparação ao primeiro. A positividade variou de $26,2 \%$ (2004) a $56,9 \%$ (2009), com valor médio de $33,8 \%$. Quanto ao tratamento químico para controle de larvas e adultos, o percentual oscilou de $27,4 \%$ (2002) e 71,1\% (2012), com média de $41,4 \%$, observando-se, para os últimos quatro anos, um aumento no percentual de imóveis tratados. Verifica-se uma tendência na diminuição do número de imóveis trabalhados ao mesmo tempo em que a positividade e o tratamento apresentam tendência de crescimento (Figura 3).

A intervenção sobre Imóveis Especiais (IE) foi inserida na norma técnica em 2002 e reiterada em 2005. A observação da Figura 4 revela que essa atividade foi realizada com certa regularidade entre 2005 e 2010, com diminuição acentuada no número dos trabalhados nos dois últimos anos, com respectiva queda da positividade, a qual oscilou de 13,5 a 37,2 com média de $24,7 \%$. O tratamento com larvicida e adulticida nesses imóveis atingiu de 19,6 a 87,6\%, com média de $37,4 \%$. Os percentuais de positividade e tratamento não apresentam tendência de crescimento (Figura 4).

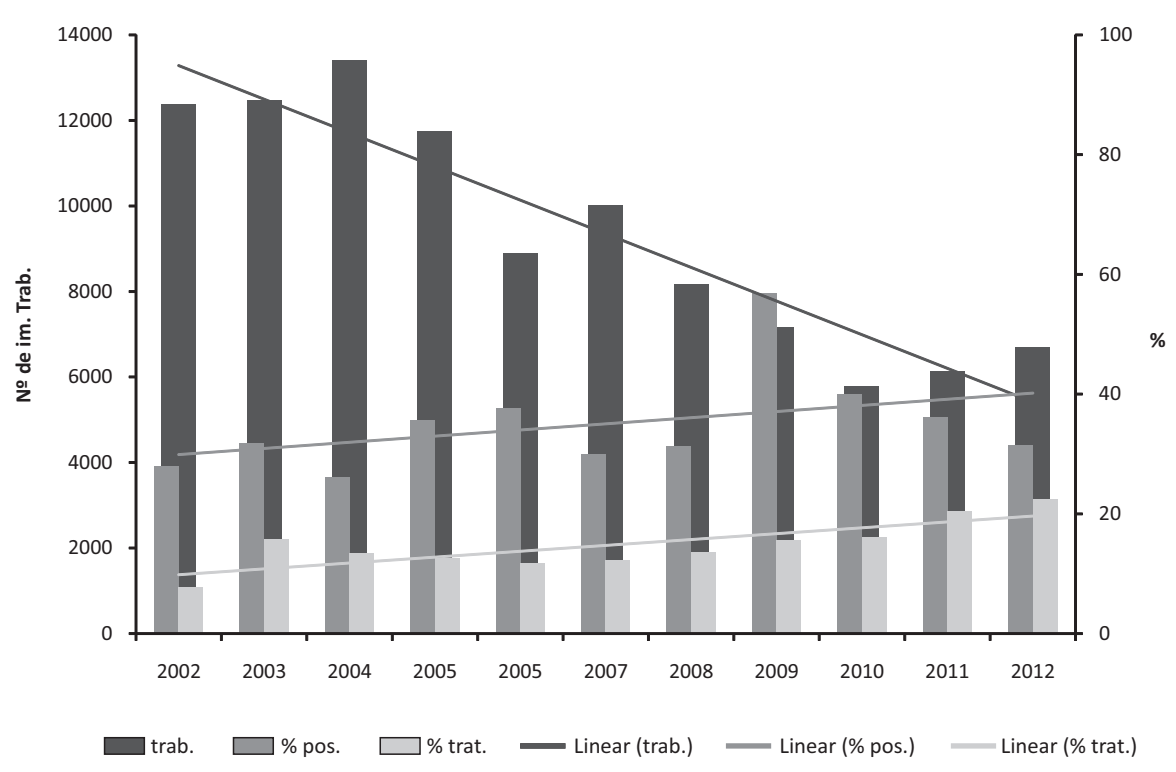

Figura 3. Número de Pontos Estratégicos trabalhados, percentual de positivos para Aedes aegypti e percentual de tratados. Municípios selecionados do Litoral Sul do Estado de São Paulo, 2002 a 2012.

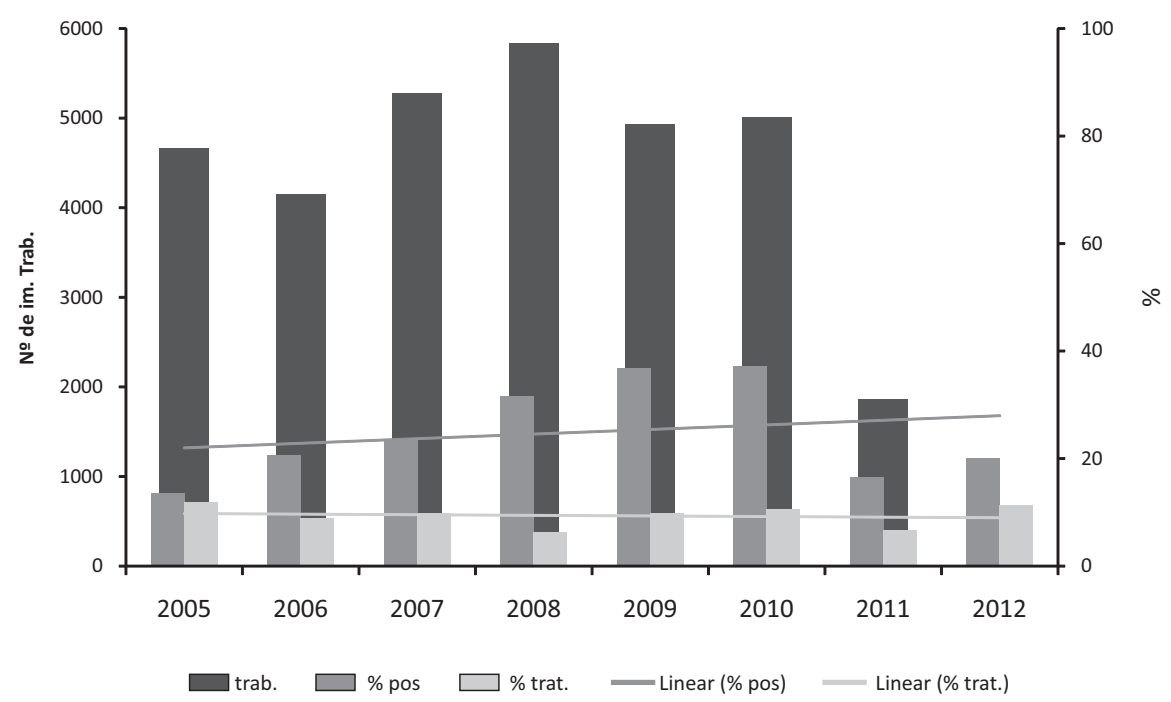

Figura 4. Número de Imóveis Especiais trabalhados, percentual de positivos para Aedes aegypti e percentual de tratados. Municípios selecionados do Litoral Sul do Estado de São Paulo, 2005 a 2012. 


\section{DISCUSSÃO}

As atividades do controle de Aedes aegypti são centradas nos criadouros potenciais do mosquito, com o emprego de condutas que visam a torná-los inadequados para a proliferação de larvas. Essas medidas podem ser físicas (eliminação, descaracterização ou destinação adequada), químicas (utilização de larvicidas) ou alternativas (não específicas), devendo a sua implementação incluir a abordagem educativa com o intuito de orientar o morador, o proprietário ou o responsável pelo imóvel a manter o ambiente impróprio para a manutenção da infestação. Devido à natureza altamente antropofílica do vetor, as atividades de seu controle são dirigidas aos locais ocupados pelo homem, principalmente nas áreas urbanas que, de modo geral, apresentam alta complexidade pela elevada concentração populacional muitas vezes com precário saneamento ambiental ${ }^{12}$.

As avaliações de índices de infestação são previstas na norma técnica do Programa de Controle do Vetor no Estado de São Paulo ${ }^{11}$, em consonância com as diretrizes nacionais $^{12}$, que preconizam medida anual no mês de outubro do Índice Predial (IP) como indicador de risco de transmissão de dengue para o ano seguinte, classificando-o em condições satisfatórias quando inferior a $1 \%$, situação de alerta, quando o valor oscilar entre $1 \%$ e $3,9 \%$ e situação de risco de transmissão de dengue para índices superiores a 3,9. Pode-se observar que o número de avaliações foi menor no mês de janeiro, com $45 \%$ das medidas realizadas, possivelmente explicadas pelo comprometimento da capacidade de trabalho em campo nas atividades de controle do vetor prioritariamente. No mês de julho, quando os índices larvários são mais baixos ${ }^{19}$ e a transmissão de dengue assume níveis residuais ${ }^{20}$, era de se esperar maior número de medidas; no entanto, apenas 12 das 20 previstas foram realizadas. É de se destacar que a vigilância entomológica é fundamental para a avaliação do impacto das medidas de controle e realização de ajustes nas ações implementadas ${ }^{21,22}$.

No período estudado, os valores do Índice Predial (IIP) nos meses avaliados, mantiveram-se abaixo de $2 \%$, porém em níveis suficientes para manter a transmissão de dengue de forma endêmica, inclusive com importante epidemia em 2010, alcançando 35.303 casos notificados (CVE), o que corrobora Taiul, quando afirma que, mesmo em baixos níveis de infestação do Aedes aegypti, é muito difícil evitar casos de dengue, pois não é possível evitar a entrada de portadores de vírus em áreas infestadas².

Em estudo anterior realizado na região, foram analisados os indicadores larvários avaliados mensalmente entre os anos de 2006 e 2011, tendo sido observado que os maiores índices de infestação de Aedes aegypti surgiram nos meses mais quentes do ano, coincidindo com o período mais favorável para a transmissão de dengue, que o comportamento do indicador no último trimestre do ano pode modular a situação da transmissão no início do ano seguinte e que o vetor está presente ao longo de todo o ano, o que facilita a transmissão na região ${ }^{23}$.

$\mathrm{Na}$ avaliação dos Pontos Estratégicos (PE), verificou-se uma grande oscilação na execução da atividade, com importante decréscimo do número de imóveis trabalhados a partir de 2005. A positividade expressiva, com média de $33,8 \%$, mostrou que os imóveis são importantes como geradores do vetor e que medidas para sua eliminação devem ser conduzidas de forma regular e contínua, com o envolvimento da vigilância sanitária, assim como de outros setores responsáveis pela regulamentação desses imóveis. Verificou-se que o tratamento químico foi empregado em número muito inferior entre os imóveis positivos para o vetor. É conhecido que, na região, os programas municipais de controle do vetor utilizaram o sal (hipoclorito de sódio) como produto alternativo para impedir o desenvolvimento de larvas. Essa conduta pode justificar o baixo percentual de imóveis com tratamento químico, condição que não é possível ser confirmada pelo sistema de informação. No entanto, a diminuição das intervenções sobre esses imóveis constitui motivo de preocupação uma vez que a sua importância se demonstra pelas informações analisadas, quando se compara o percentual de PE positivos e IIP obtidos. Esses imóveis com acúmulo de recipientes (PE) podem manter a infestação na área e dificultar a diminuição da infestação vetorial ${ }^{19}$.

A atividade de Imóveis Especiais (IE), estabelecida em 2002, somente foi incorporada de forma sistemática a partir de 2005 , quando se verificou que, ao longo do período estudado, o número de visitas não apresentou grandes oscilações, com aumento nos anos de 2011 e 2012. A média de positividade da atividade é significativa (24,7\%), e o baixo percentual de tratamentos pode ter a mesma explicação já referida para os Pontos Estratégicos. A importância desses imóveis já foi demonstrada por estudo realizado na própria região que comparou as taxas de infestação para formas imaturas de Aedes aegypti obtidas nesses imóveis com outros de menor porte, mostrando serem estes bem maiores, reforçando sua importância em receber cuidados especiais para evitar a presença do vetor. Também foi reiterada em análise de indicadores coletados em todo Estado a recomendação de que as ações de vigilância e controle vetorial precisam ser implementadas nesses e que, por serem geralmente, mais trabalhosas e complexas que em outros imóveis, devem ser cadastrados para trabalho em atividade específica, para melhor monitoramento ${ }^{19}$. Os Imóveis Especiais (IE) representam grande importância no controle de dengue, principalmente durante o período de transmissão da doença, devido ao maior risco de circulação do vírus pela função que executam, tais como unidades de assistência à saúde, escolas, clubes, entre outros. Seu controle deve contar com a participação efetiva dos seus responsáveis, por meio da criação do Núcleo de Prevenção à Dengue (NPD) formado por funcionários capacitados, acompanhados pelas equipes municipais de controle de vetores, possibilitando, assim, melhor resultado da ação. 
A atividade de casa-a-casa que abrange a maioria dos imóveis de uma área infestada pelo vetor, sendo expressos principalmente por domicílios, apresentou cobertura irregular, com variação de $50 \%$ até $140 \%$. Uma cobertura mais baixa no primeiro quadrimestre do ano pode ser justificada pela transmissão de dengue, observada principalmente em 2010, ano em que a região apresentou grande número de casos de dengue, sendo o recurso humano direcionado para as atividades de controle. Por meio dos indicadores obtidos nos sistemas de informação vigentes e de acordo com a situação epidemiológica do município, a intensificação da atividade de casa-a-casa tem sido planejada e realizada de forma a garantir a priorização das áreas problemáticas. No entanto, carece de melhor explicação a baixa realização desta atividade em 2012.

O presente estudo mostrou a dificuldade de operacionalização das atividades de rotina propostas na norma técnica ${ }^{11}$, havendo descontinuidade em seu cumprimento, baixas coberturas em todas as atividades, sem que possa ser reconhecido o direcionamento do recurso existente para as atividades prioritárias para o controle da infestação pelo vetor da dengue (PE) e diminuição do risco de disseminação do vírus (IE). Também não foi possível constatar a completa implantação das orientações técnicas no que se refere às avaliações entomológicas, originadas pelas informações coletadas (IIP), como subsídio às estratégias de redução da infestação em áreas prioritárias. Quanto às visitas casa-a-casa, a orientação de realização de dois ciclos quadrimestrais por ano, com intensificação nas áreas mais problemáticas, pareceu estar implantada na região; no entanto, carece aprofundar a análise local visando garantir a qualidade das ações executadas para impactar a redução da infestação constituindo obstáculo à transmissão. Este aspecto tem sido apontado como determinante para o comprometimento da eficácia das ações de controle do vetor da dengue ${ }^{2,25}$.

Particularmente para municípios de grande porte, o alcance das metas estabelecidas de redução de índices de infestação larvária poderia ser efetivado por ação mais focalizada em locais com presença de vetores previamente detectada, além de maior rigidez no controle daqueles imóveis que oferecem condições favoráveis para a reprodução vetorial, como prevê a norma em vigor no Estado de São Paulo ${ }^{11,26}$

\section{REFERÊNCIAS}

1. Braga IM. Aedes aegypti: histórico do controle no Brasil. Epidemiol Serv Saúde. 2007;16(2):113-118.

2. Tauil PL. Aspectos críticos do controle do dengue no Brasil. Cad Saúde Pública. 2002, 18(3):867-871. doi: http://dx.doi.org/10.1590/S0102$311 \times 2002000300035$.

3. Ministério da Saúde (BR) (Fundação Nacional de Saúde). Programa Nacional de Controle da Dengue (PNCD) [Internet]. Brasília: Funasa; 2002 [acesso em 10 Jan 2013]. 32 p. Disponível em: http://bvsms. saude.gov.br/bvs/publicacoes/pncd_2002.pdf.

4. Glasser CM, Gomes AC. Infestação do Estado de São Paulo por Aedes aegypti e Aedes albopictus. Rev Saúde Pública [Internet]. 2000 Dez [acesso em 07 Ago 2013];34(6):570-577. Disponível em: http://www.scielosp.org/scielo.php?script=sci_arttext\&pid=S0034$89102000000600002 \&$ lng=en. doi: http://dx.doi.org/10.1590/S003489102000000600002 .

5. Glasser CM, Pereira M, Katz G, Kavakama BB, Souza LTM, Ferreira IB, Rocco IM, Alves MCGP. Dengue no Estado de São Paulo: exemplo da complexidade do problema neste final de século. Rev CIP. 1999;2(4):11-20.

6. Departamento de Informática do SUS - DATASUS (BR), Sistema de Informação de Agravos de Notificação - SINAN, Centro de Vigilância Epidemiológica - CVE. [base de dados na Internet]. São Paulo: Divisão de Zoonoses do Estado do Centro de Vigilância Epidemiológica do Estado de São Paulo. 2008 - Casos autóctones de dengue por DIR e Município no Estado de São Paulo - 1995 a 2006; [acesso em 12 Abr 2013]. Disponível em: http://www.cve.saude.sp.gov.br/htm/zoo/Den_ dir06.htm.

7. Ministério da Saúde (BR). Portaria no. 1399, de 15 de dezembro de 1999. Regulamenta a NOB SUS $01 / 96$ no que se refere às competências da União, Estados, Municípios e Distrito Federal, na área de epidemiologia e controle de doenças, define a sistemática de financiamento e dá outras providências [Internet]. 1999 [acesso em 12 Abr 2013]. Disponível em: http://www.funasa.gov.br/site/wpcontent/files_mf/Pm_1399_1999.pdf.

8. Ministério da Saúde (BR). Portaria no. 1172, de 15 de junho de 2004. Regulamenta a NOB SUS 01/96 no que se refere às competências da União, Estados, Municípios e Distrito Federal, na área de Vigilância em Saúde, define a sistemática de financiamento e dá outras providências [Internet]. 2004 [acesso em 12 Abr 2013]. Disponível em: http:// dtr2001.saude.gov.br/sas/PORTARIAS/Port2004/GM/GM-1172.htm.

9. Ministério da Saúde (BR). Portaria no. 3252, de 22 de dezembro de 2009. Aprova as diretrizes para execução e financiamento das ações de Vigilância em Saúde pela União, Estados, Distrito Federal e Municípios e dá outras providências [Internet]. 2009 [acesso em 12 Abr 2013]. Disponível em: http://portal.saude.gov.br/portal/arquivos/pdf/ portaria3252_da_vigilancia_em_saude_0501_atual.pdf.

10. Ministério da Saúde (BR). Portaria no. 1378, de 9 de julho de 2013. Regulamenta as responsabilidades e define diretrizes para execução e financiamentos das ações de Vigilância em Saúde pela União, Estados, Distrito Federal e Municípios, relativos ao Sistema Nacional de Vigilância em Saúde e Sistema Nacional de Vigilância Sanitária [Internet]. 2013 [acesso em 15 Jul 2013]. Disponível em: http:// www.renastonline.org/blog/olgarios/portaria-13782013-substituiportaria-32522009

11. Secretaria de Estado da Saúde de São Paulo (BR) (Superintendência de Controle de Endemias - SUCEN). Normas e Recomendações Técnicas para a Vigilância e Controle de Aedes aegypti no Estado de São Paulo. São Paulo: SUCEN; 2005. 108 p.

12. Ministério da Saúde (BR) (Secretaria de Vigilância em Saúde, Departamento de Vigilância Epidemiológica). Diretrizes nacionais para a prevenção e controle de epidemias de dengue. Brasília: Ministério da Saúde; 2009. 160 p. 
13. Secretaria de Estado da Saúde (BR) (Superintendência de Controle de Endemias - SUCEN). Normas e Recomendações Técnicas para a Vigilância e Controle de Aedes aegypti no Estado de São Paulo. São Paulo: SUCEN; 2002. $70 \mathrm{p}$.

14. Instituto Brasileiro de Geografia e Estatística - IBGE (BR). Censo Demográfico 2000-2010 [Internet]. Brasília: IBGE. 2010 [acesso em 12 Abr 2013]. Disponivel em: http://www.censo2010.ibge.gov.br/ apps/mapa/.

15. Portal Brasil. Geografia: Tipos de clima [Internet]. Brasília: Governo Federal; (C2010 [acesso em 06 Ago 2013]. Disponível em: http:// www.brasil.gov.br/sobre/meio-ambiente/geografia/tipos-de-clima.

16. Governo do Estado de São Paulo (BR) (Secretaria de Planejamento e Desenvolvimento Regional). Caracterização socioeconômica de São Paulo - Região Metropolitana da Baixada Santista. São Paulo: Governo do Estado de São Paulo; 2011. 66 p.

17. Secretaria de Estado da Saúde de São Paulo (BR) (Coordenadoria de Controle de Doenças, Superintendência de Controle de Endemias). Programa de Vigilância e Controle da Dengue [Internet]. São Paulo: SUCEN; 2010. 65 p. Disponível em: http://www.saude.sp.gov. $\mathrm{br} /$ resources/sucen/homepage/downloads/arquivos-dengue/ programa_estadual.pdf.

18. Holcman MM, Barbosa GL, Andrade VR, Domingos MF, Gomes AHA, Silva M, Wanderley DMV. Infestação por Aedes aegypti em imóveis fechados nas visitas para vigilância e controle vetorial de dengue em municípios do Estado de São Paulo. Bol Epidemiol Paulista [Internet]. 2012 [acesso em 12 Abr 2013];9(104):14-23. Disponível em: http:// www.cve.saude.sp.gov.br/bepa/txt/bepa104_aedes.htm.

19. Barbosa GL, Holcman MM, Pereira M, Gomes AHA, Wanderley DMV. Indicadores de infestação larvária e influência do porte populacional na transmissão de dengue no estado de São Paulo, Brasil: um estudo ecológico no período de 2007-2008. Epidemiol Serv Saúde 2012;21(2):195-204. doi: http://dx.doi.org/10.5123/S167949742012000200002

20. Secretaria de Estado da Saúde de São Paulo (BR) (Coordenadoria de Controle de Doenças - CCD, Centro de Vigilância Epidemiológica CVE, Superintendência de Controle de endemias - SUCEN, Instituto Adolfo Lutz). Informe Técnico [Dengue], de 05 de Junho de 2011. Identificação do sorotipo DENV 4, GVE 29 - São José do Rio Preto [Internet]. 2011 [acesso em 12 Abr 2013]. Disponível em: http:// www.cve.saude.sp.gov.br/htm/zoo/pdf/if11_dengue1005.pdf.
21. Donalísio MR, Glasser CM. Vigilância entomológica e controle de vetores do dengue. Rev Bras Epidemiol [Internet]. 2002 [acesso em 12 Abr 2013];5(3):259-279. Disponível em: http://www.scielo.br/scielo. php?script=sci_arttext\&pid=S1415-790X2002000300005\&lng=en . doi: http://dx.doi.org/10.1590/S1415-790X2002000300005.

22. Gomes AC. Vigilância entomológica. Inf Epidemiol SUS [Internet]. 2002 [acesso em 12 Abr 2013];11(2):79-90. Disponível em: http:// scielo.iec.pa.gov.br/scielo.php?script=sci_arttext\&pid=S0104$16732002000200004 \&$ Ing=pt\&nrm=iso.

23. Domingos MF, Losano NF, Carvalho M, Wanderley DMV. Larval indices of Aedesagypti and dengue transmission in endemic region of São Paulo State, Brazil. In: XVIII International Congress for Tropical Medicine and Malaria and XLVIII Congress of the Brazilian Society of Tropical Medicine; 2012; Rio de Janeiro: Sociedade Brasileira de Medicina Tropical; 2012.

24. Glasser $\mathrm{CM}$, Arduino MB, Barbosa GL, Ciaravolo RMC, Domingos MF, Oliveira CD, Pereira M, Silva M, Trevisan AMY. Comportamento de formas imaturas de Aedes aegypti no litoral do Estado de São Paulo. Rev Soc Bras Med Trop [Internet]. 2011 [acesso em $12 \mathrm{Abr}$ 2013];44(3):349-355. Disponível em: http://www.scielo.br/scielo. php?script=sci_arttext\&pid=S0037-86822011000300018\&lng=en. doi: http://dx.doi.org/10.1590/\$0037-86822011005000042.

25. Wermelinger ED, Cohen SR, Thaumaturgo C, Silva, AA, Ramos FAF, Souza MB. Avaliação do acesso aos criadouros do Aedes aegypti por agentes de saúde do programa de Saúde da Família no município do Rio de Janeiro. Rev Baiana Saúde Pública. 2008;32(2):151-158.

26. Pessanha JEM, Caiaffa WT, César CC, Proietti FA. Avaliação do Plano Nacional de Controle de Dengue. Cad Saúde Pública. Jul 2009;25(7):1637-1641. doi: http://dx.doi.org/10.1590/S0102311X2009000700024. PubMed PMID: 19578587.

Como citar este artigo / How to cite this article:

Wanderley DMV. Atividades de controle de Aedes aegypti em municípios da região sul do Estado de São Paulo. J Health Biol Sci. 2013 Jul-Set; 1(3):122-129. 